\title{
Structural and Electronic Properties of the SiC (100) Surfaces
}

\author{
J. S. Soares and H. W. Leite Alves \\ Departamento de Ciências Naturais, Universidade Federal de São João del Rei, \\ Caixa Postal 110, 36300-000 São João del Rei-MG, Brazil
}

Received on 4 April, 2005

\begin{abstract}
In this work, we present our preliminary ab initio results for the structural and electronic properties of both $\mathrm{Si}$ - and C-terminated $\mathrm{SiC}(100)$ surfaces in $(2 \times 1)$ and $\mathrm{c}(2 \times 2)$ reconstruction patterns. Based on our results, we found that the Si-terminated surfaces are dominated by weak bonded Si-dimers, which are stabilized only at Si-rich conditions, leading to $(3 \times 2)$ or more complex reconstruction patterns, as verified experimentally. Also, our results show that the $\mathrm{C}$-terminated surfaces is characterized by strong triply-bonded $\mathrm{C}$-dimers, in a $\mathrm{c}(2 \times 2)$ reconstruction pattern, which consists of $\mathrm{C}_{2}$ pairs over Si bridge sites, in agreement with experimental results.
\end{abstract}

Keywords: SiC (100); Ab initio results; Structural and electronic properties

\section{INTRODUCTION}

$\mathrm{SiC}$ is the most indicated material for the high temperature semiconductor devices, besides the fact that this compound is the most promising substrate for the epitaxial growth of IIINitrides. In this specific growth, the most common substrate surface used is the (100) one [1,2].

It is well known that (100) surfaces have a large number of reconstruction patterns of a large degree of multiplicity and complexity [3] and, for the $\mathrm{SiC}$ (100) surfaces, this is also the case $[4,5]$.

So, there has been a great interest for their understanding, in order to improve the epitaxial growth of both the III-Nitrides, and the bulk SiC for high-temperature and highpower devices. For instance, while the Si-terminated surfaces are stable with the $(3 \times 2)$ or $(2 \times 1)$ reconstruction patterns only at Si-rich conditions, the C-terminated ones, which is known by its chemical inertness and thermal stability, have an interesting arrangement of $\mathrm{c}(2 \times 2) \mathrm{C}$-dimers, with each dimer consisting with a triple bond among the $\mathrm{C}$ atoms [5].

In order to understand the complexity of the reconstructions of $\mathrm{SiC}$ surfaces, we present in this work, a preliminary comparative study of the geometry, bond behaviour, the energetics, as well as the electronic structure of the $(2 \times 1)$ reconstruction model of the Si-terminated surfaces, and of the $(2 \times 1)$ and $c(2 \times 2)$ reconstruction patterns of the C-terminated ones. Our results were obtained by using the Density Functional Theory (DFT) within the Local Density Approximation (LDA), plane-wave description of the wavefunctions and the pseudopotential method (abinit code [6]). We have used the Troullier-Martins pseudopotentials (fhi98PP code [7]), following the recipe developed by Stampfl and Van de Walle [8]. The $\mathrm{C}(\mathrm{Si})$-terminated surfaces were described by slab supercells build up of 6(5) atomic layers and a vacuum region equivalent of 4(5) atomic layers, and the back surface was passivated by hydrogen atoms. For the slab lattice parameters, we have used our calculated equilibrium bulk lattice parameter for cubic SiC, $4.31 \AA$, obtained with a cutoff energy of $35 \mathrm{H}$ (70 Ry) for the plane-wave expansion and 10 special k-points. Also, in the slab calculations, a (4 4 2) Monkhorst-Pack set of k-points was used to sample the slab Brillouin zone [9], and we kept, for the plane-wave expansion, the same cutoff energy of the converged bulk results.

\section{SI-TERMINATED SURFACES}

In Table 1, we display the structural parameters for the Siterminated $(2 \times 1)$ surfaces, as defined in Fig. 1. It is natural to expect that the $\mathrm{Si}$-dimers rearrange themselves in this reconstruction pattern once that, for the $\mathrm{Si}(100)$ surfaces, they are described within the $\mathrm{p}(2 \times 1)$ or $\mathrm{c}(4 \times 2)$ models [10]. However, based on our results, we found that the Si-terminated $\mathrm{SiC}$ surfaces is dominated by weak bonded unbuckled Si-dimers, once the dimer length is greater than the experimental value. It is surprising because, compared with the $\mathrm{Si}$ surfaces, the Siterminated SiC surfaces are around $20 \%$ compressed. Moreover, our results are in good agreement with other LDA-based calculations, since there are some other theoretical results that contradict this picture [5].

When we increase the surface coverage of $1 / 2$ monolayer by adding a $\mathrm{Si}$ atom, leading to $\mathrm{c}(4 \times 2)$ pattern, besides the fact that the new system became $10 \mathrm{meV} /$ atom more stable than the previous $(2 \times 1)$ pattern, the dimer length diminish toward the experimental value. This is an indication that the Siterminated $\mathrm{SiC}$ surfaces are stable only at Si-rich conditions, leading to Si-satured surfaces in a complex reconstruction pattern, such as $(3 \times 2)$, as verified experimentally [11].

TABLE I: Calculated structural parameters, as defined in Fig. 1, for the Si-terminated $\mathrm{SiC}(100)$ surfaces in the $\mathrm{p}(2 \times 1)$ reconstruction model and with an extra Si adatom.

\begin{tabular}{|l|l|l|}
\hline Parameter & $\mathrm{p}(2 \times 1)$ & $\mathrm{p}(2 \times 1)+\mathrm{Si}$ \\
\hline$\alpha($ degrees $)$ & 108.57 & 108.28 \\
\hline$\beta($ degrees $)$ & 93.54 & 97.29 \\
\hline $\mathrm{Z}(\AA)$ & -0.03 & -0.02 \\
\hline $\mathrm{A}(\AA)$ & 2.80 & 2.65 \\
\hline $\mathrm{A}_{\exp }(\AA)[5]$ & 2.31 & 2.31 \\
\hline $\mathrm{B}(\AA)$ & 1.88 & 1.98 \\
\hline $\mathrm{C}(\AA)$ & 3.05 & 3.21 \\
\hline $\mathrm{D}(\AA)$ & 3.04 & 3.15 \\
\hline
\end{tabular}




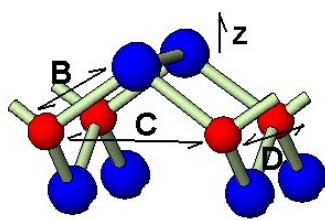

(a)

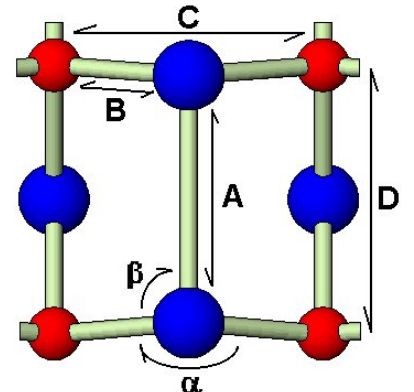

(b)

FIG. 1: Structural parameters which describes the atomic geometry of the SiC (100) surfaces. (a) Perspective view of the surface dimer and the two sub-surface layers. (b) Top view of the surface dimer and the two sub-surface layers.

In Fig. 2, we depicted the calculated electronic band structure for the $(2 \times 1)$ pattern of the Si-terminated surfaces. Our results show that, at $\bar{\Gamma}$ point of the Brillouin zone, there are two states in the bandgap, one at $\mathrm{E}_{v}+0.59 \mathrm{eV}$ with $\pi^{*}$ character, fully occupied, and the other at $\mathrm{E}_{v}+0.93 \mathrm{eV}$ with $\sigma$ character, totally unoccupied, and are in agreement with other LDA calculations, and with both EELS and XPS data [5]. These energy levels have similar features to both the highest occupied molecular orbital and the lowest unoccupied molecular orbital of the $\mathrm{Si}_{2}$ molecule, respectively. However, the $\sigma$ states have higher energies than the $\pi$ ones, instead. This is due to the following aspects: i) the Si-terminated $\mathrm{SiC}$ (100) surfaces are $20 \%$ smaller than the Si (100) ones and strained Si-dimers are found; ii) carbon is more electronegative than silicon and the latter behave as a cation at the surface with different hybridization of that observed in the Si (100) surface, with a strong contribution from the Si- $\mathrm{p}_{z}$ orbitals [12]. Moreover, we have observed that the energy difference between $\pi$ and $\pi^{*}$ levels, $1.04 \mathrm{eV}$, remains almost constant for all wave vectors of the first Brillouin zone, as already observed in previous calculations $[5,12]$.

\section{C-TERMINATED SURFACES}

It is well known that, for the C-terminated surfaces, there are two competing models that explain the observed $\mathrm{c}(2 \times 2)$ reconstruction, the Bridge-Dimer Model (BDM) and the Staggered-Dimer Model (SDM), which is directly related to the two possible preparation methods for this surface [5]. In Table II we display the structural parameters for the reconstructed surfaces as defined in Fig. 1, and we have verified that our results are in good agreement with other previous theoretical and experimental results $[4,5]$.

From Table II, we checked that both bond angles, $\alpha$ and $\beta$, tend to 120 degrees, indicating that each $\mathrm{C}$ dimer atom tries to make planar bonds with the other one. Also, it is interesting to note that, in both the $(2 \times 1)$ and the SDM patterns, the

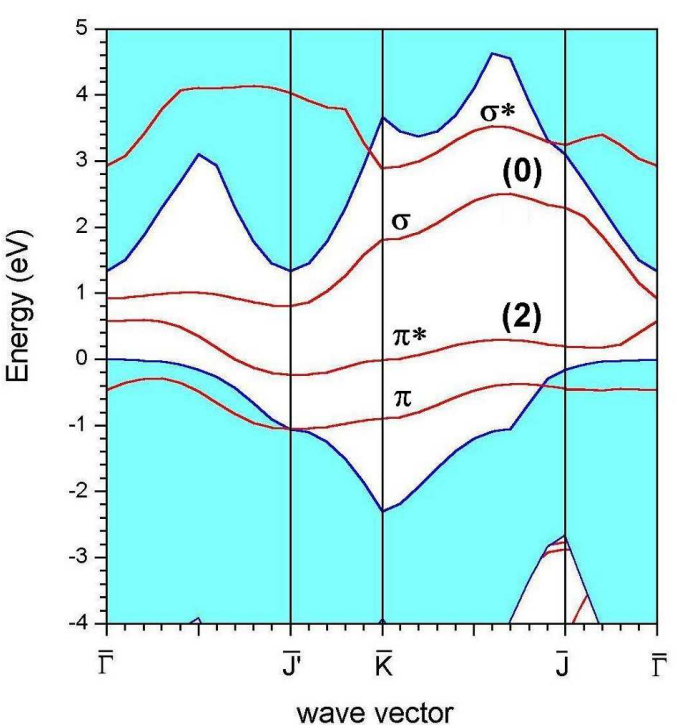

FIG. 2: Calculated bands of Si-terminated SiC $(100)(2 \times 1)$ surface. The number in parenthesis indicates the population of the highest occupied level and of the lowest unoccupied level, while the hatched area are the projected bulk results.

surface atoms move inward, while in the BDM, the surface relaxes in the opposite way. This is a clear indication that the $\mathrm{C}-\mathrm{C}$ bonds, in the latter model, have different character when compared with the previous one. Moreover, if we look at the dimer bond length (parameter A), in both the $(2 \times 1)$ and the $\mathrm{SDM}$ it approaches to the $\mathrm{C}-\mathrm{C}$ double bond, as verified for the ethylene, while in the BDM, it is close to the $\mathrm{C}-\mathrm{C}$ triple bond of the acetylene. So, our results are in consonance with previous total energy calculations [5,13], and, also, confirm that the C-terminated surface is completely dependent of its preparation method.

TABLE II: Calculated structural parameters, as defined in Fig.1, for the C-terminated $\mathrm{SiC}(100)$ surfaces in the $\mathrm{p}(2 \times 1), \mathrm{c}(2 \times 2) \mathrm{SDM}$ and $\mathrm{c}(2 \times 2) \mathrm{BDM}$ reconstruction patterns depicted in Fig. 1 . The datum in the parenthesis of the last column is the experimental result for the Si-dimer bond length in the BDM, which is the fingerprint of this specific model, taken from Ref. 5.

\begin{tabular}{|l|l|l|l|}
\hline Parameter & $\mathrm{p}(2 \times 1)$ & $\mathrm{c}(2 \times 2)$ SDM & $\mathrm{C}(2 \times 2) \mathrm{BDM}$ \\
\hline$\alpha($ degrees $)$ & 112.02 & 105.64 & - \\
\hline$\beta($ degrees $)$ & 110.77 & 115.94 & 133.00 \\
\hline $\mathrm{z}(\AA)$ & -0.29 & -0.20 & 0.21 \\
\hline $\mathrm{A}(\AA)$ & 1.43 & 1.37 & 1.23 \\
\hline $\mathrm{A}_{\text {exp }}(\AA)[5]$ & 1.31 & 1.31 & 1.22 \\
\hline $\mathrm{B}(\AA)$ & 1.84 & 1.91 & 1.82 \\
\hline $\mathrm{C}(\AA)$ & 3.05 & 3.05 & 3.12 \\
\hline $\mathrm{D}(\AA)$ & 2.73 & 3.05 & $2.39(2.70)$ \\
\hline
\end{tabular}

It is also interesting to note, from Table II, that there is a $\mathrm{Si}$ dimer at the sub-surface in the BDM, once the value for the parameter $\mathrm{D}$ is $2.39 \AA$, and this is a fingerprint of this specific 
model.

From the total energy point of view, the BDM is 7.0 meV/atom more stable than SDM, in agreement with the theoretical results of Käckell et al. [13]. However, the BDM is only favored by $2 \mathrm{meV} /$ atom over the $(2 \times 1)$ reconstruction pattern. Based on the fact that our calculations have the accuracy of $3 \mathrm{meV}$, and that the total energy differences between these three models are small, we can deduce that these patterns can coexist at high temperatures.

When we look at the calculated electronic band structures of these models, our results show that while for the $(2 \times 1)$ pattern, it has a metallic character, for both the BDM and SDM they have a semiconductor character instead. In Figs. 3 and 4, we depicted the calculated electronic band structure for both the BDM and the SDM models, respectively.

From Fig. 3, we found that, at $\bar{\Gamma}$ point of the Brillouin zone, there are two states in the bandgap, one at $\mathrm{E}_{v}+0.84$ $\mathrm{eV}$, and the other at $\mathrm{E}_{v}+0.50 \mathrm{eV}$, both fully occupied. While the first one has the main contribution from the Si sub-surface atom, remaining in the gap with a small dispersion of $0.67 \mathrm{eV}$, the second one has a strong $\mathrm{C}$-dimer $\pi$-bond character, as that observed on the $\mathrm{C}-\mathrm{C}$ triple bonds in the acetylene molecule. The latter has a strong dispersion, of around $1.5 \mathrm{eV}$, so that it leaves the gap at the $\bar{M}$ point. Also, the first unoccupied level, which is resonant at the conduction band at $\mathrm{E}_{v}+3.58 \mathrm{eV}$ in $\bar{\Gamma}$, has a $\pi$ antibonding character, and a flat dispersion, lying in the bandgap at the $\bar{M}$ point.

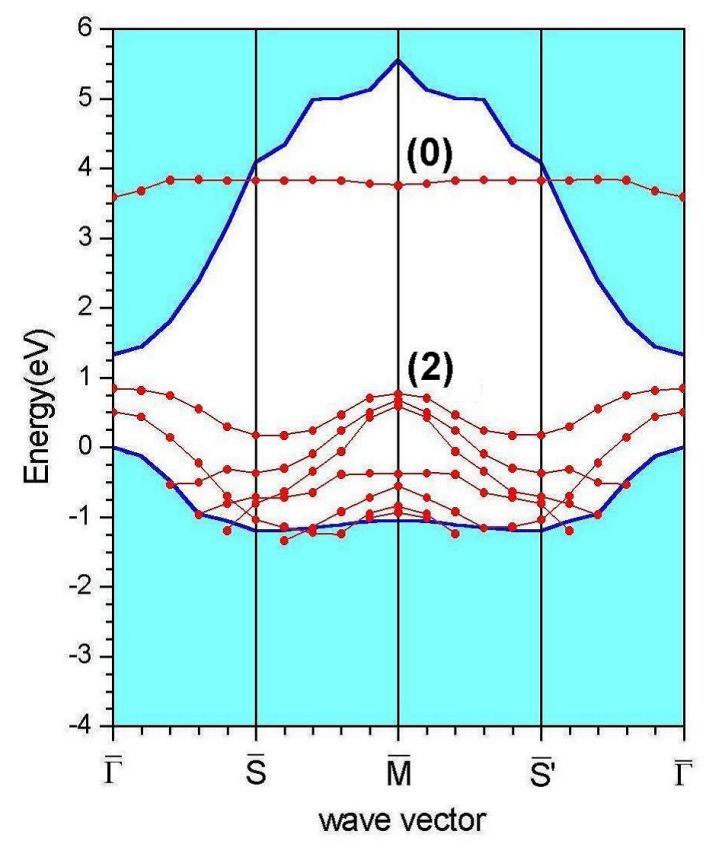

FIG. 3: Same as Fig. 2 for the C-terminated SiC (100) c $(2 \times 2)$ BDM surface.

From Fig. 4, we detected that the calculated band structure of the SDM has almost the same appearance of that of BDM. However, instead of two (as in the BDM case), there are three occupied levels in the bandgap at $\bar{\Gamma}$ point of the Brillouin zone. The highest occupied level, located at $\mathrm{E}_{v}+0.97$ $\mathrm{eV}$, has the same character of the obtained highest occupied level for BDM, while the lowest unoccupied, which lies at $\mathrm{E}_{v}$ $+2.41 \mathrm{eV}$, is also resonant at the conduction band, but has 1.0 $\mathrm{eV}$ of dispersion and has the same antibonding character of the molecular orbitals which describe the $\mathrm{C}-\mathrm{C}$ double bonds in the ethylene molecule.

\section{FINAL REMARKS}

In summary, we have studied the geometry, bond behaviour, the energetics as well as the electronic structure for the $(2 \times 1)$ reconstruction model of the Si-terminated surfaces, and for $(2 \times 1)$ and $c(2 \times 2)$ reconstruction patterns within both BDM and SDM models of the C-terminated ones. We found that the $\mathrm{C}$-terminated surfaces are characterized by strong triplybonded $\mathrm{C}$-dimers, in the $\mathrm{c}(2 \times 2)$ reconstruction pattern, consisting of $\mathrm{C}_{2}$ pairs over $\mathrm{Si}$ bridge sites. Also, we show that the Si-terminated surfaces are dominated by weak bonded $\mathrm{Si}$ dimers, which at Si-rich conditions, are Si-satured surfaces in a $(3 \times 2)$ or more complex reconstruction patterns.

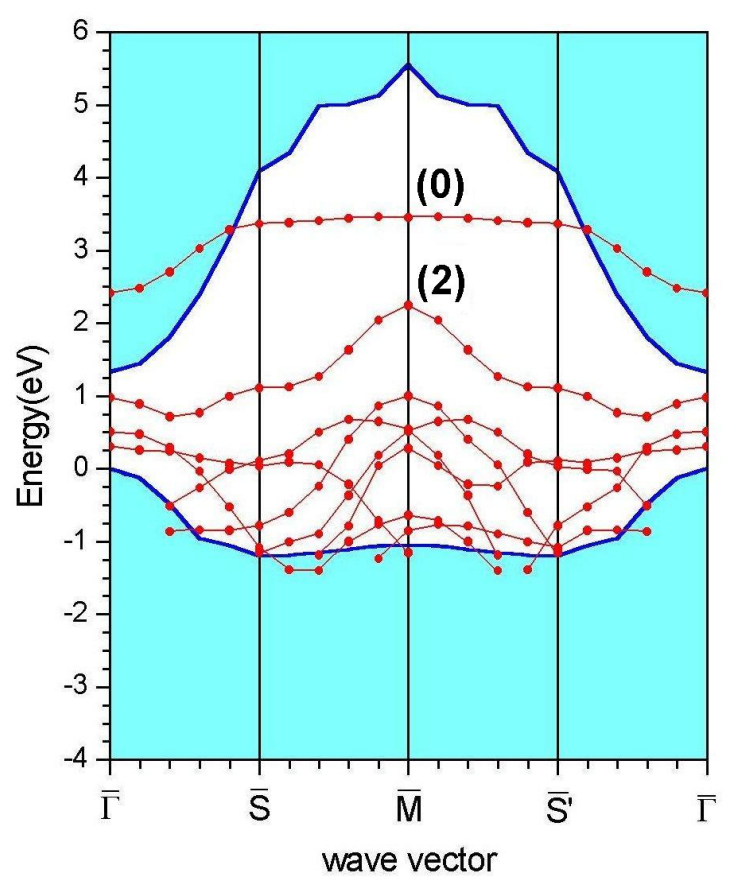

FIG. 4: Same as Fig. 2 for the C-terminated SiC (100) c $(2 \times 2)$ SDM surface.

\section{Acknowledgements}

J. S. Soares was supported by the Brazilian Program PIBIC/CNPq. This work was also supported by the MCT/CNPq/PRONEX project No. 662105/98, Brazil. 
[1] R. S. Kern and R. F. Davies, Mater. Sci. Eng. B46, 240 (1997).

[2] S. C. Jain, M. Willander, J. Narayan, and R. van Overstraeten, J. Appl. Phys. 87, 965 (2000).

[3] A. Kahn, Surf. Rev. Lett. 3, 1579 (1996).

[4] V. M. Bermudez, Phys. Stat. Sol. (b)202, 447 (1997), and references therein.

[5] J. Pollmann and P. Krüger, J. Phys.: Condens. Matter 16, S1659 (2004), and references therein.

[6] X. Gonze et al., Comput. Mater. Sci. 25, 478 (2002), and references therein.

[7] M. Fuchs and M. Scheffler, Comput. Phys. Commun. 119, 67 (1999), and references therein.

[8] C. Stampfl and C. G. Van de Walle, Phys. Rev. B 59, 5521
(1999).

[9] H. J. Monkhorst and J. D. Pack, Phys. Rev. B13, 5188 (1976).

[10] J. Ihm, D. H. Lee, J. D. Joannopoulos, and J. J. Xiong, Phys. Rev. Lett. 51, 1872 (1983); J. Fritsch and P. Pavone, Surf. Sci. 344, 159 (1995).

[11] P. Soukiassian, Mater. Sci. Eng. B96, 115 (2002); V. Derycke et al., Nature Materials 2, 253 (2003).

[12] M. Sabisch, P. Krüger, A. Mazur, M. Rohlfing, and J. Pollmann, Phys. Rev. B 53, 13121 (1996).

[13] P. Käckell, J. Furthmüller, F. Bechstedt, G. Kresse, and J. Hafner, Phys. Rev. B44, 10304 (1996). 